\title{
Changes in plasma lipids predict pravastatin efficacy in secondary prevention
}

\author{
Kaushala S. Jayawardana, ${ }^{1}$ Piyushkumar A. Mundra, ${ }^{1}$ Corey Giles, ${ }^{1}$ Christopher K. Barlow, ${ }^{1}$ \\ Paul J. Nestel, ${ }^{1}$ Elizabeth H. Barnes, ${ }^{2}$ Adrienne Kirby, ${ }^{2}$ Peter Thompson, ${ }^{3}$ David R. Sullivan, ${ }^{4}$ \\ Zahir H. Alshehry, ${ }^{1,5}$ Natalie A. Mellett, ${ }^{1}$ Kevin Huynh, ${ }^{1}$ Malcolm J. McConville, ${ }^{6}$ Sophia Zoungas, ${ }^{7,8}$ \\ Graham S. Hillis, ${ }^{7,9}$ John Chalmers, ${ }^{7}$ Mark Woodward, ${ }^{710}$ Ian C. Marschner, ${ }^{2,11}$ Gerard Wong, ${ }^{1}$ \\ Bronwyn A. Kingwell, John Simes, ${ }^{2}$ Andrew M. Tonkin, ${ }^{8}$ and Peter J. Meikle, ${ }^{1,6,8}$ \\ on behalf of the LIPID Study Investigators ${ }^{12}$
}

${ }^{1}$ Baker Heart and Diabetes Institute, Melbourne, Victoria, Australia. ${ }^{2}$ National Health and Medical Research Council of Australia (NHMRC) Clinical Trials Centre, University of Sydney, Sydney, New South Wales, Australia. ${ }^{3}$ Sir Charles Gairdner Hospital, Perth, Western Australia, Australia. ${ }^{4}$ Royal Prince Alfred Hospital, Sydney, New South Wales, Australia. ${ }^{5}$ King Fahad Medical City, Riyadh, Saudi Arabia. ${ }^{6}$ Department of Biochemistry and Molecular Biology, University of Melbourne, Parkville, Victoria, Australia. ${ }^{7}$ The George Institute for Global Health, Sydney, New South Wales, Australia. ${ }^{8}$ Monash University, Melbourne, Victoria, Australia. ${ }^{9}$ The Royal Perth Hospital and University of Western Australia, Perth, Western Australia, Australia. ${ }^{10}$ The George Institute for Global Health, University of Oxford, England. "Department of Mathematics and Statistics, Macquarie University, Sydney, New South Wales, Australia. ${ }^{12}$ The LIPID Study Investigators are detailed in the Supplemental Acknowledgments.

BACKCROUND. Statins have pleiotropic effects on lipid metabolism. The relationship between these effects and future cardiovascular events is unknown. We characterized the changes in lipids upon pravastatin treatment and defined the relationship with risk reduction for future cardiovascular events.

METHODS. Plasma lipids ( $n=342$ ) were measured in baseline and 1-year follow-up samples from a Long-Term Intervention with Pravastatin in Ischaemic Disease (LIPID) study subcohort $(n=4991)$. The associations of changes in lipids with treatment and cardiovascular outcomes were investigated using linear and Cox regression. The effect of treatment on future cardiovascular outcomes was examined by the relative risk reduction (RRR).

RESULTS. Pravastatin treatment was associated with changes in 206 lipids. Species containing arachidonic acid were positively associated while phosphatidylinositol species were negatively associated with pravastatin treatment. The RRR from pravastatin treatment for cardiovascular events decreased from $23.5 \%$ to $16.6 \%$ after adjustment for clinical risk factors and change in LDLcholesterol (LDL-C) and to $3.0 \%$ after further adjustment for the change in the lipid ratio $\mathrm{PI}(36: 2)$ / $\mathrm{PC}(38: 4)$. Change in $\mathrm{PI}(36: 2) / \mathrm{PC}(38: 4)$ mediated $58 \%$ of the treatment effect. Stratification of patients into quartiles of change in $\mathrm{PI}(36: 2) / \mathrm{PC}(38: 4)$ indicated no benefit of pravastatin in the fourth quartile.

Authorship note: $\mathrm{KS}$ ), PAM, and CG are co-first authors.

Conflict of interest: PJM has licensed lipid biomarkers to Zora Biosciences. A patent relating to the method of predicting drug therapeutic responder status and methods of treatment has been filed (PCT/AU2018/051371)

Copyright: (c) 2019, American Society for Clinical Investigation.

Submitted: February 27, 2019

Accepted: May 22, 2019

Published: July 11, 2019.

Reference information: JCI Insight. 2019;4(13):e128438. https://doi. org/10.1172/jci.insight.128438.
CONCLUSION. The change in $\mathrm{PI}(36: 2) / \mathrm{PC}(38: 4)$ predicted benefit from pravastatin, independent of change in LDL-C, demonstrating its potential as a biomarker for monitoring the clinical benefit of statin treatment in secondary prevention.

TRIAL REGISTRATION. Australian New Zealand Clinical Trials Registry identifier ACTRN12616000535471.

FUNDING. Bristol-Myers Squibb; NHMRC grants 211086, 358395, and 1029754; NHMRC program grant 1149987; NHMRC fellowship 108026; and the Operational Infrastructure Support Program of the Victorian government of Australia. 


\section{Introduction}

Reduction in LDL-cholesterol (LDL-C) is thought to be the major mechanism by which statins reduce the risk of cardiovascular events in primary prevention $(1,2)$. In the Long-Term Intervention with Pravastatin in Ischaemic Disease (LIPID) study (3), which recruited patients who were stable after a previous acute coronary syndrome, change in LDL-C was found to be an important mechanism of treatment action. However, findings in the LIPID study (4) and in other studies $(5,6)$ suggest that further mechanisms, in addition to change in LDL-C, might also be important to fully explain the statin treatment effect and to identify poor responders to statin treatment.

Plasma lipid classes, subclasses, and species have been shown to associate with cardiovascular events and cardiovascular deaths and have prognostic value independent of traditional risk factors in both primary and secondary prevention populations (7-11). HMG-CoA reductase inhibitors (statins) have been extensively prescribed to patients to control their cholesterol and LDL-C levels, but these medications also change levels of several other plasma lipid classes and individual species $(12,13)$. We hypothesize that some of the benefits of pravastatin could be mediated by this effect and that early changes in these same lipid species will identify those who are more likely to benefit from statin treatment (i.e., receive a relative risk reduction $[R R R])$.

In this manuscript, we report on the largest single lipidomic study to date; we analyze 342 lipid species in 4991 participants at 2 time points. We first investigated the relationship between pravastatin treatment and the change in lipid species from baseline to 1-year follow-up. In a landmark analysis, we assessed associations of the change in lipid species with future cardiovascular events and cardiovascular deaths independent of changes in LDL-C levels. Further, we investigated the risk reduction associated with changes in lipid species over a period of 1 year. Finally, using a mediation analysis, we investigated the possible causal relationship of these changes with cardiovascular outcomes.

\section{Results}

Baseline characteristics. The LIPID cohort analyzed in this study consisted of 4991 participants with a median age of 64 years (quartile 1 to quartile 3 [Q1-Q3], 57-69 years). Plasma samples were collected at both baseline and the 1-year follow-up. In this cohort, 944 patients experienced cardiovascular events after the 1-year follow-up, and there were 498 cardiovascular deaths (Supplemental Figure 1; supplemental material available online with this article; https://doi.org/10.1172/jci.insight.128438DS1). Details of baseline characteristics stratified by outcome in the present subcohort are shown in Supplemental Table 1. Individuals who experienced cardiovascular events in the follow-up period were older, had higher blood pressure, and were more likely to have a history of diabetes, stroke, and atrial fibrillation compared with individuals who did not have events. Importantly, with the exception of HDL-cholesterol (HDL-C), the clinical lipid measures (LDL-C and triglycerides) were not significantly different between the groups that did or did not experience cardiovascular events or cardiovascular death.

Changes to the plasma lipidome upon pravastatin treatment. Comparisons of the mean percentage change in lipid classes/subclasses and species in the first year of pravastatin treatment between placebo and pravastatin treatment arms are shown in Supplemental Tables 2 and 3. As expected, with the exception of phosphatidylserine, all lipid classes/subclasses were reduced by pravastatin treatment, and the majority of lipid species were significantly reduced in the pravastatin group relative to the placebo group. There were only 8 lipid species that showed an increase in the pravastatin group relative to the placebo group. These were predominately characterized by the presence of arachidonic acid (20:4) or its precursor, dihomo- $\gamma$-linolenic acid (20:3), in 6 of 8 of these lipid species (Supplemental Table 3).

In assessing the relationship between pravastatin treatment and percentage change in lipid classes and subclasses, independent of age, sex, BMI, and the percentage changes in total cholesterol, HDL-C, and triglycerides, 17 of the 24 lipid classes/subclasses were significantly associated with treatment after correction for multiple comparisons (Supplemental Table 4). Most of the significantly associated lipid classes/subclasses showed a negative association with treatment, indicating that these classes were decreased by pravastatin independently of the changes in clinical lipids (total cholesterol, HDL-C, and triglycerides). Dihydroceramide and lysophosphatidylinositol showed a positive association with treatment after adjustment for changes in clinical lipids but still showed a decrease in absolute levels (Supplemental Table 2). Of the 342 lipid species examined, 206 were significantly associated with treatment after correction for multiple comparisons (Figure 1 and Supplemental Table 5). 


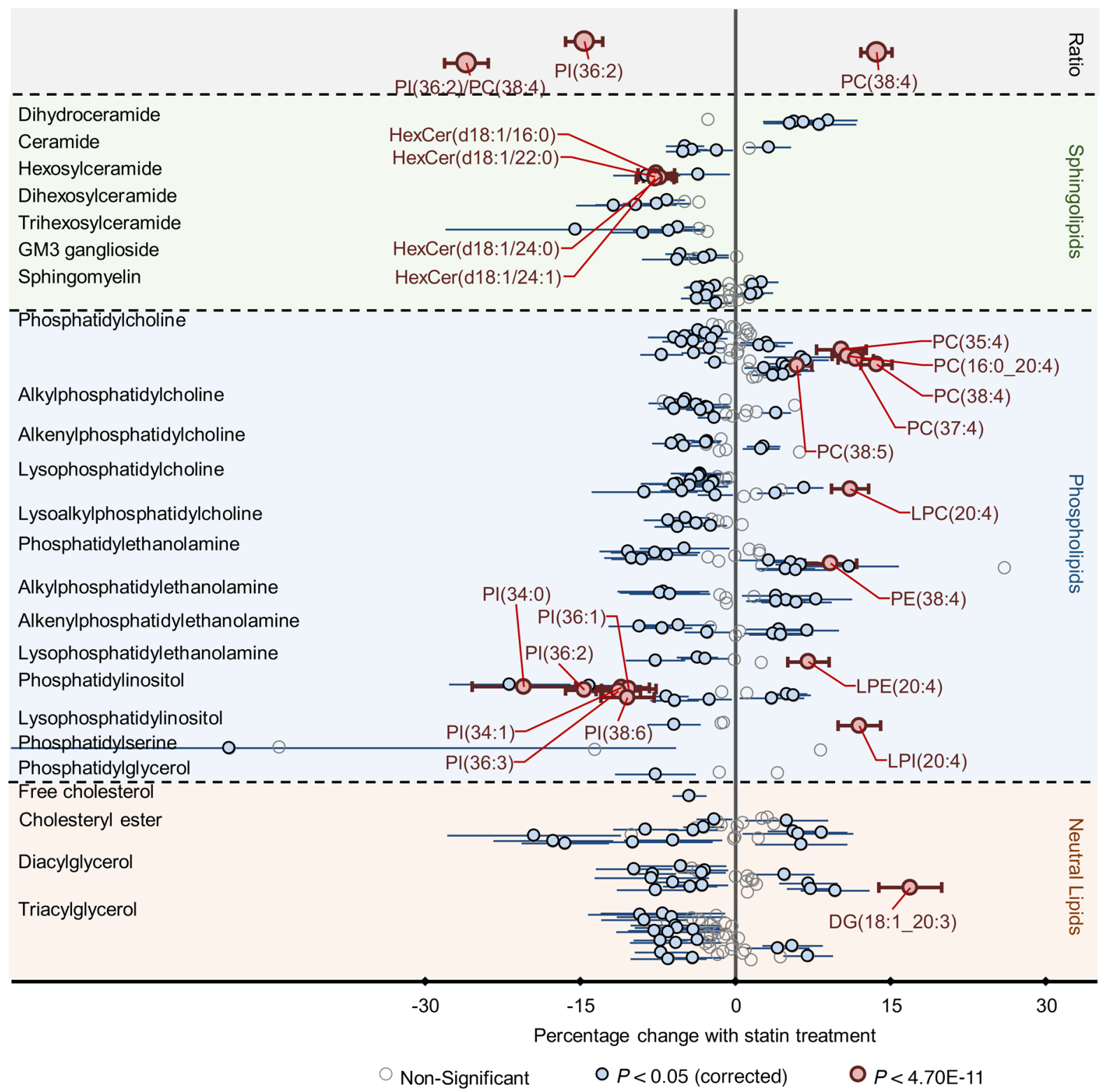

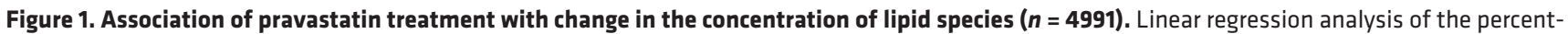
age change in each lipid species against statin treatment, adjusted for age, sex, BMI, and percentage change in cholesterol, HDL-C, and triglycerides, was performed. The $\beta$-coefficient denotes the difference in percentage changes (from baseline to follow-up) between treatment and placebo groups. DG, diacylglycerol; HexCer, monohexosylceramide; LPC, Iysophosphatidylcholine; LPE, Iysophosphatidylethanolamine; LPI, Iysophosphatidylinositol; PC, phosphatidylcholine; PI, phosphatidylinositol.

There were 138 lipid species that showed a negative association with statin treatment independent of changes in clinical lipids. Eleven of the sixteen phosphatidylinositol species were negatively associated with pravastatin treatment, three species were positively associated, and two species were not significantly associated. Of the 50 phosphatidylcholine species measured, 12 were negatively associated, while 18 were positively associated with treatment.

To validate these findings, we also performed regression analysis of statin treatment against the plasma lipid concentrations at the 1-year time point (LIPID study) and at baseline (Action in Diabetes and Vascular Disease: Preterax and Diamicron-MR Controlled Evaluation [ADVANCE] study), adjusting for age, sex, BMI, total cholesterol, HDL-C, and triglycerides. The associations observed at the 1-year point in the LIPID study (Figure 2) were very similar to those observed for the percentage change in lipid species (Figure 1). Similarly, the associations observed at baseline in the ADVANCE study were very similar to those observed in the LIPID study (Figure 2 and Supplemental Table 6). 


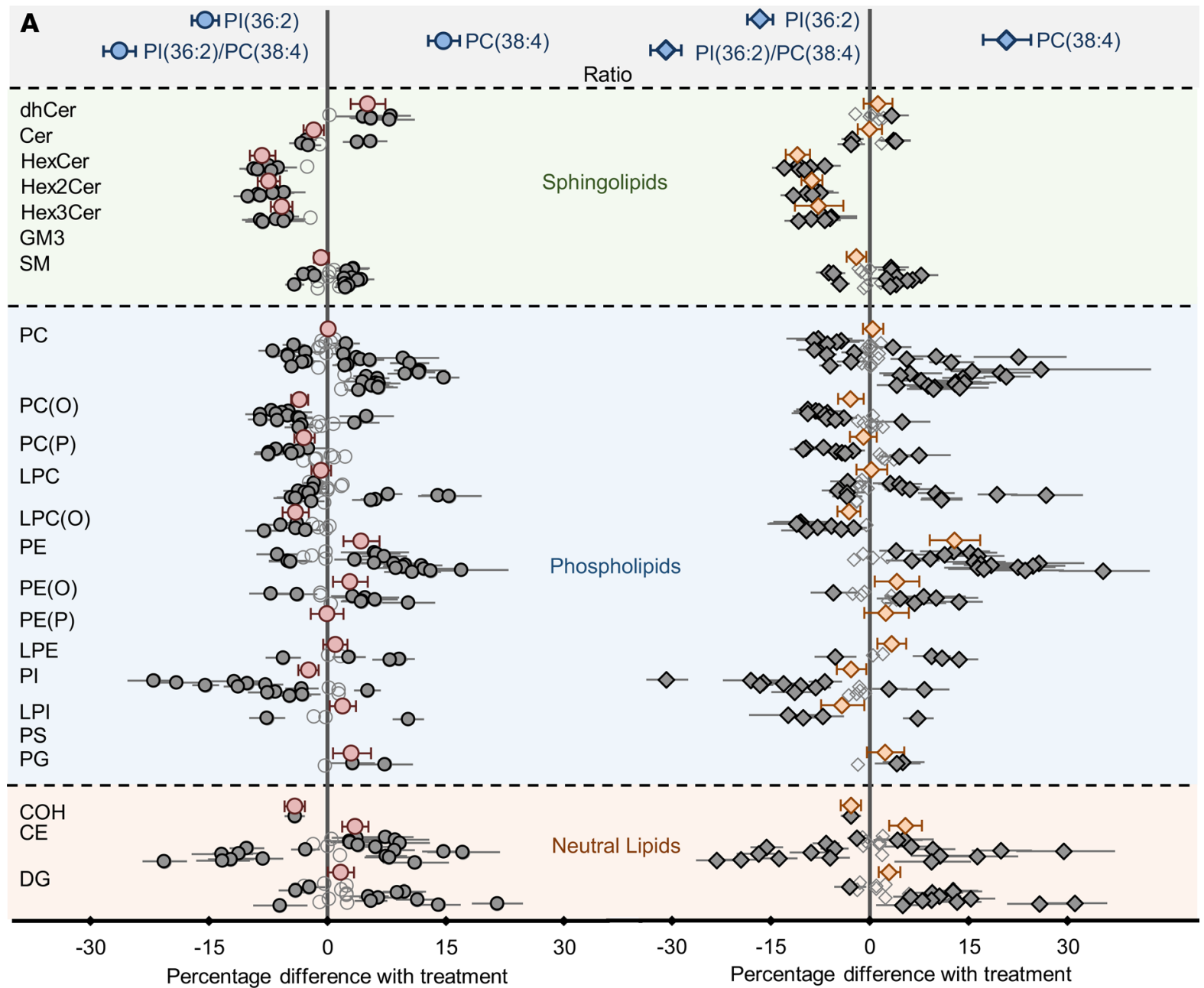

Non-Significant $O P<0.05$ (corrected) OLipid class LIPID study

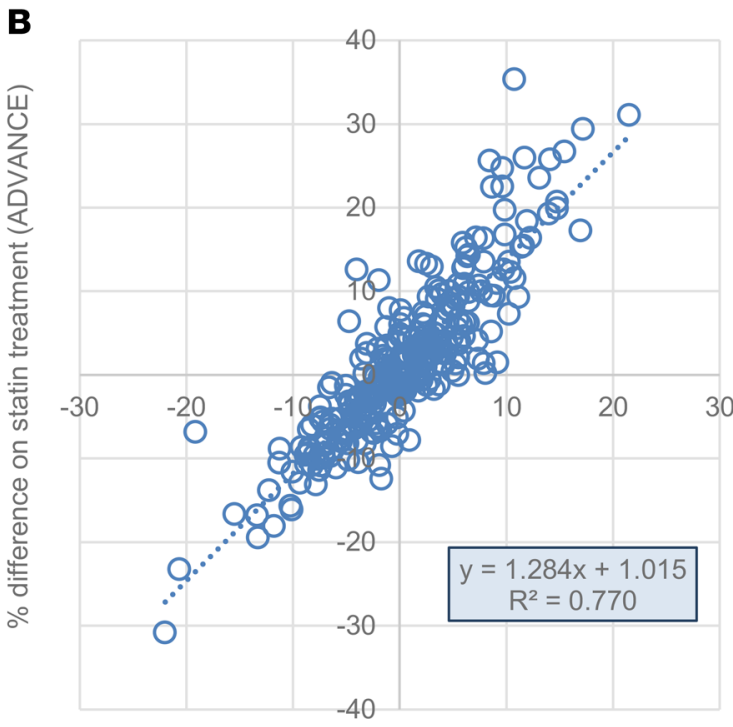

$\%$ difference on statin treatment (LIPID) $\diamond$ Non-Significant $\diamond P<0.05$ (corrected) $\diamond$ Lipid class

ADVANCE study

Figure 2. Association of statin treatment with percentage difference of lipid species and classes. (A) Percentage difference between statin-treated and untreated participants of the LIPID study (taken at the 12-month time point; $n=4991$ ) and the ADVANCE study (taken at baseline; $n=3779$ ). The percentage difference values were analyzed against statin treatment in a linear regression analysis, adjusted for age, sex, BMI, cholesterol, HDL-C, and triglycerides. (B) Correlation between the percentage difference between statin-treated and untreated participants in the LIPID study and ADVANCE study as analyzed via a linear regression. $\mathrm{CE}$, cholesteryl ester; Cer, ceramide; $\mathrm{COH}$, free cholesterol; DG, diacylglycerol; dhCer, dihydroceramide; $\mathrm{CM} 3, \mathrm{G}_{\mathrm{M}}$ ganglioside; HexCer, monohexosylceramide; Hex2Cer, dihexosylceramide; Hex3Cer, trihexosylceramide; LPC, lysophosphatidylcholine; LPC(O), lysoalkylphosphatidylcholine; LPE, Iysophosphatidylethanolamine; LPI, lysophosphatidylinositol; PC, phosphatidylcholine; $P C(0)$, alkylphosphatidylcholine; $P C(P)$, alkenylphosphatidylcholine; $\mathrm{PE}$, phosphatidylethanolamine; $\mathrm{PE}(\mathrm{O})$, alkylphosphatidylethanolamine; $\mathrm{PE}(\mathrm{P})$, alkenylphosphatidylethanolamine; $\mathrm{PG}$, phosphatidylglycerol; PI, phosphatidylinositol; PS, phosphatidylserine; SM, sphingomyelin; TG, triacylglycerol. 


\begin{tabular}{|c|c|c|c|c|c|c|c|}
\hline \multirow[b]{2}{*}{ Base } & \multicolumn{3}{|c|}{$\begin{array}{l}\text { Adjusted for lipid } \\
\text { species }\end{array}$} & \multirow{2}{*}{$\begin{array}{c}\text { Hazard ratio } \\
(95 \% \mathrm{Cl})\end{array}$} & \multirow{2}{*}{$\begin{array}{l}P \text { value } \\
4.55 \mathrm{E}-05 \\
\end{array}$} & \multirow{2}{*}{$\begin{array}{l}\text { RRR } \\
23.5 \% \\
\end{array}$} & \multirow[t]{2}{*}{$\begin{array}{l}P \text { value } \\
\text { for change } \\
\text { in } \operatorname{RRR}^{1}\end{array}$} \\
\hline & - & $\longmapsto \infty$ & & & & & \\
\hline Base + change in LDL-C & - & $\longmapsto$ & & $0.834(0.700,0.993)$ & $4.19 \mathrm{E}-02$ & $16.6 \%$ & \\
\hline \multirow{11}{*}{$\begin{array}{l}\text { Base + change in LDL-C + change in lipid } \\
\text { species + baseline lipid levels + interaction } \\
\text { between change in lipid and treatment }\end{array}$} & $\mathrm{PC}\left(16: 0 \_20: 4\right)$ & $-\infty$ & ب & $0.878(0.734,1.050)$ & $1.54 \mathrm{E}-01$ & $12.2 \%$ & $1.00 \mathrm{E}-04$ \\
\hline & $\mathrm{Pl}(32: 0)$ & $\vdash$ & - & $0.878(0.736,1.048)$ & $1.49 \mathrm{E}-01$ & $12.2 \%$ & $3.63 \mathrm{E}-02$ \\
\hline & $\operatorname{Pl}(34: 1)$ & & -1 & $0.878(0.738,1.046)$ & $1.45 \mathrm{E}-01$ & $12.2 \%$ & $2.83 \mathrm{E}-02$ \\
\hline & $\mathrm{Pl}(38: 6)$ & & $\longrightarrow$ & $0.880(0.737,1.051)$ & 1.60E-01 & $12.0 \%$ & $4.06 \mathrm{E}-02$ \\
\hline & $\operatorname{LPC}(16: 0)$ & & $\longrightarrow$ & $0.881(0.736,1.055)$ & 1.69E-01 & $11.9 \%$ & 1.93E-02 \\
\hline & $P C\left(18: 2 \_20: 4\right)$ & $\vdash$ & $\longrightarrow$ & $0.891(0.746,1.064)$ & $2.01 \mathrm{E}-01$ & $10.9 \%$ & $1.00 \mathrm{E}-04$ \\
\hline & $\operatorname{LPC}(20: 4)$ & & $\longrightarrow$ & $0.895(0.748,1.070)$ & $2.24 \mathrm{E}-01$ & $10.5 \%$ & $1.00 \mathrm{E}-04$ \\
\hline & $\mathrm{PC}(38: 4)$ & & $\longrightarrow$ & $0.907(0.758,1.085)$ & 2.87E-01 & $9.3 \%$ & 1.00E-04 \\
\hline & $\mathrm{PI}(36: 3)$ & & $\longrightarrow$ & $0.909(0.761,1.085)$ & 2.92E-01 & $9.1 \%$ & 3.70E-03 \\
\hline & PI(36:2) & $\longmapsto$ & $\square$ & $0.920(0.765,1.105)$ & $3.72 \mathrm{E}-01$ & $8.0 \%$ & 2.18E-02 \\
\hline & $\mathrm{PI}(36: 2) / \mathrm{PC}(38: 4)$ & $\mapsto$ & $\longrightarrow$ & $0.970(0.807,1.166)$ & 7.46E-01 & $3.0 \%$ & 7.00E-04 \\
\hline
\end{tabular}

Figure 3. Association of treatment with future cardiovascular events after adjustment for changes in LDL-C and lipid concentration ( $n=4991)$. The base model describes the association of treatment with future cardiovascular events analyzed via Cox regression, adjusted for the Marschner covariates (age, sex, total cholesterol, HDL-C, current smoking, nature of prior acute coronary syndrome, revascularization, diabetes history, stroke history, and history of hypertension). The subsequent models incorporate change in LDL-C and change in plasma lipid species into the model with base covariates described above. The hazard ratios and relative risk reduction (RRR) are for those individuals who did not exhibit a change in the lipid species. The hazard ratios adjusted for the interaction effect between the change in lipid species and treatment depend on the magnitude of the change in lipid species. LPC, lysophosphatidylcholine; PC, phosphatidylcholine; PI, phosphatidylinositol.

Association of change in lipid species concentration with future events within the treatment group. Cox regression analyses, adjusted for baseline risk factors and the baseline values of each lipid species, show that change in 50 and 35 lipid species were associated with future cardiovascular events and cardiovascular death, respectively $(P<0.05$, uncorrected). However, these associations were attenuated after correction for multiple comparisons, with the exception of 6 species with future cardiovascular events (Supplemental Figure 3 and Supplemental Table 7). A majority of the phosphatidylinositol species showed changes that were positively associated with future events, indicating that a greater decrease in these lipids was associated with fewer cardiovascular events and deaths. In contrast, the phospholipid species containing arachidonic acid (20:4) had changes that were negatively associated, indicating that an increase - relative to changes in clinical lipids - in these lipid species was associated with fewer adverse cardiovascular events. The change in the lipid ratio, consisting of a phosphatidylinositol species [PI(36:2)] divided by a phosphatidylcholine species [PC(38:4)] to give $\mathrm{PI}(36: 2) / \mathrm{PC}(38: 4)$, showed a highly significant positive association with both future cardiovascular events and cardiovascular death, indicating that a larger decrease in the ratio was associated with fewer cardiovascular events and deaths. In contrast, when the analyses were repeated in the placebo group, the change in lipid ratio showed no association with either future cardiovascular events or cardiovascular death (Supplemental Table 7). Furthermore, a test of interaction between treatment and change in lipid ratio was statistically significant in each case $(P=1.27 \mathrm{E}-02$ and $P=4.16 \mathrm{E}-02$, respectively). These results suggest a moderating effect of change in the lipid ratio.

The change in plasma lipid species influences the pravastatin treatment effect. The effect of pravastatin treatment on reduction in cardiovascular events and cardiovascular death before and after adjustment for the change in LDL-C ( $\triangle$ LDL-C) and changes in concentration of lipid species are shown in Figure 3 and Supplemental Tables 8 and 9. After adjusting for the 10 Marschner covariates (age, sex, total cholesterol, HDL-cholesterol, current smoking, nature of prior acute coronary syndrome, revascularization, diabetes history, stroke history, history of hypertension), pravastatin was associated with a $23.5 \%$ RRR in cardiovascular events and $21.6 \% \mathrm{RRR}$ in cardiovascular deaths. After further adjustment for $\triangle \mathrm{LDL}-\mathrm{C}$, the estimated RRRs by pravastatin were $16.6 \%$ and $17.4 \%$ for cardiovascular events and cardiovascular deaths, respectively. When examining cardiovascular events as the outcome and further adjusting for the changes in individual lipid species, baseline lipid values, and the interaction between changes in lipid species and treatment, RRR was reduced to $8.0 \%$ for individuals with no reduction in $\mathrm{PI}(36: 2)$. For those with a reduction in $\mathrm{PI}(36: 2)$, treatment provided a greater RRR (interaction effect $P=1.50 \mathrm{E}-02$, Supplemental Table 8). Without adjusting for $\triangle \mathrm{LDL}-\mathrm{C}$, the estimated decrease in cardiovascular events by pravastatin was reduced from $23.5 \%$ to $12.2 \%$ in individuals with no change in $\mathrm{PI}(36: 2)$. The change in $\mathrm{PC}(38: 4)$ also resulted in a 
Table 1. Association of statin treatment with future cardiovascular events or cardiovascular death

\begin{tabular}{|c|c|c|c|c|c|c|}
\hline \multirow[b]{2}{*}{ Outcome } & \multirow[b]{2}{*}{$\begin{array}{l}\text { Hazard ratio }(95 \% \\
\text { CI) }\end{array}$} & \multirow[b]{2}{*}{$P$ value } & \multicolumn{2}{|c|}{ Adjusted for $\Delta L^{B}$} & \multicolumn{2}{|c|}{ Adjusted for $\Delta L D L-C^{c}$} \\
\hline & & & $\begin{array}{l}\text { Hazard ratio }(95 \% \\
\mathrm{Cl})\end{array}$ & $P$ value & $\begin{array}{l}\text { Hazard ratio (95\% } \\
\mathrm{Cl})\end{array}$ & $P$ value \\
\hline \multicolumn{2}{|c|}{ Cardiovascular death $0.784(0.657,0.936)$} & 7.15E-03 & $0.905(0.735,1.114)$ & $3.45 \mathrm{E}-01$ & $0.825(0.613,1.109)$ & $2.02 \mathrm{E}-01$ \\
\hline
\end{tabular}

significantly lower RRR after adjusting for $\triangle \mathrm{LDL}-\mathrm{C}$ (reduced from $16.6 \%$ to $9.3 \%$ in individuals with no lipid species response), while this RRR was greater with an increase in $\mathrm{PC}(38: 4)$ level. However, the interaction effect was not statistically significant in this case $(P=1.87 \mathrm{E}-01)$.

Similar trends were observed with cardiovascular death. In individuals with no lipid species response, the RRR decreased from $17.4 \%$ to $7.8 \%$ and $8.1 \%$ when the models were adjusted for the change in PI(36:2) and PC(38:4), respectively. Results for all the lipid species are shown in Supplemental Tables 8 and 9. These results indicate that the benefit of treatment is highly diminished when a lipid species response is absent.

Landmark analysis using the lipid ratio. The changes in the lipid species $\mathrm{PI}(36: 2)$ and $\mathrm{PC}(38: 4)$ showed significant associations with pravastatin treatment (negative and positive associations, respectively) and in the opposite directions with future cardiovascular outcomes (positive and negative associations, respectively). In Cox regression models, the changes in these species also resulted in significant decreases in the RRR compared with the RRR resulting from models where only the change in LDL-C was included.

The change in the lipid ratio $\mathrm{PI}(36: 2) / \mathrm{PC}(38: 4)(\Delta \mathrm{LR})$ was significantly associated with pravastatin treatment independent of age, sex, BMI, and the changes in total cholesterol, HDL-C, and triglycerides (Supplemental Table 5). Although the individual lipid species PI(36:2) and PC(38:4) showed negative and positive associations with pravastatin treatment, respectively, the ratio of these lipid species showed a stronger negative association. The $\Delta \mathrm{LR}$ was significantly associated with future cardiovascular events and cardiovascular death, independent of baseline risk factors, including $\triangle \mathrm{LDL}-\mathrm{C}$ and baseline values of the lipid ratio (Supplemental Table 7). This association was positive, indicating that a larger decrease in this lipid ratio was associated with fewer cardiovascular events and deaths.

The addition of the $\Delta \mathrm{LR}$, baseline lipid ratio, and interaction term for $\Delta \mathrm{LR}$ and treatment to the base model with $\triangle \mathrm{LDL}-\mathrm{C}$ reduced the RRR to $3.0 \%$ in individuals with no change in $\triangle \mathrm{LR}$ (Figure 3 and Supplemental Table 8). Without the adjustment for $\triangle \mathrm{LDL}-\mathrm{C}, \mathrm{RRR}$ was reduced to $8.5 \%$ in such individuals (base model RRR $=23.5 \%$ ). For cardiovascular death, the addition of $\triangle$ LDL-C lowered the RRR from $21.6 \%$ to $17.4 \%$. The addition of $\Delta \mathrm{LR}$ further decreased the RRR to $1.0 \%$ in individuals with no change in $\Delta \mathrm{LR}$ (Supplemental Table 9). The individuals who experienced a decrease in the lipid ratio exhibited greater RRR.

Causal mediation analysis. Statin treatment was associated with cardiovascular events and cardiovascular death after adjusting for Marschner covariates (Table 1). Similarly, statin treatment was associated with $\Delta \mathrm{LR}$ and $\triangle \mathrm{LDL}-\mathrm{C}$ after adjusting for Marschner covariates (Table 2). When we controlled for $\triangle \mathrm{LR}$, the association

Table 2. Association of statin treatment with $\Delta$ LR and LDL-C

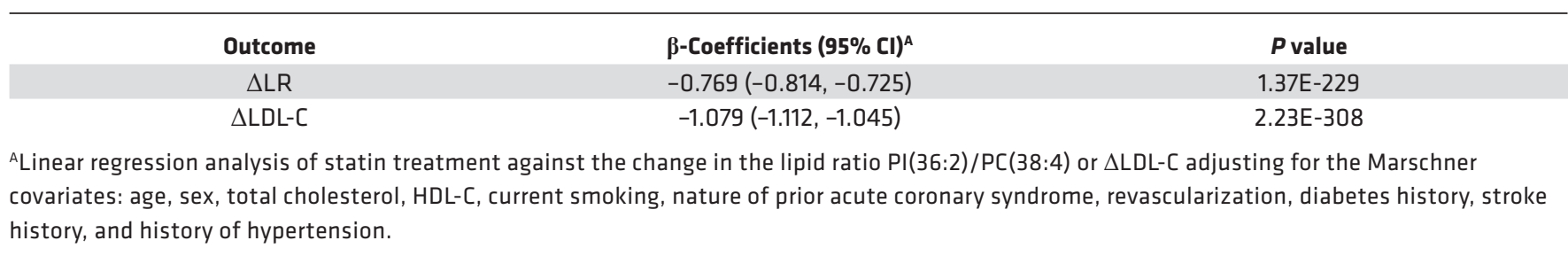


Table 3. Mediation analysis of statin treatment on cardiovascular events and cardiovascular death by the $\Delta L R$

\begin{tabular}{|c|c|c|c|c|}
\hline Effect $^{A}$ & Estimate $(95 \% \mathrm{CI})$ & $P$ value & Estimate (95\% CI) & $P$ value \\
\hline ACME $^{B}$ (placebo) & $-0.009(-0.092,0.070)$ & $8.27 \mathrm{E}-01$ & $0.029(-0.089,0.150)$ & $6.23 \mathrm{E}-01$ \\
\hline $\operatorname{ADE}^{\mathrm{C}}$ (placebo) & $-0.113(-0.265,0.040)$ & 1.37E-01 & $-0.096(-0.309,0.120)$ & 3.93E-01 \\
\hline $\mathrm{ADE}^{\mathrm{C}}$ (treated) & $-0.261(-0.420,-0.110)$ & $<1.00 E-03$ & $-0.276(-0.489,-0.060)$ & $1.24 \mathrm{E}-02$ \\
\hline Total effect & $-0.270(-0.404,-0.150)$ & $<1.00 E-03$ & $-0.247(-0.425,-0.070)$ & 7.20E-03 \\
\hline Interaction test & $-0.147(-0.283,-0.027)$ & 1.74E-02 & $-0.179(-0.361,-0.003)$ & 4.70E-02 \\
\hline \multicolumn{5}{|c|}{$\begin{array}{l}\text { AThe Marschner covariates (age, sex, total cholesterol, HDL-cholesterol, current smoking, nature of prior acute coronary syndrome, revascularization, } \\
\text { diabetes history, stroke history, and history of hypertension) were used in the analysis. }{ }^{\mathrm{B}} \text { The average causally mediated effect (the indirect effect } \\
\text { through the mediator). }{ }^{\circ} \text { The average direct effect (the direct effect of the statin treatment). }{ }^{D} \text { The proportion of the total effect facilitated by the } \\
\text { mediator. The bold values indicate significant } P \text { values }(P<0.05) \text {. }\end{array}$} \\
\hline
\end{tabular}

of statin treatment with cardiovascular events or cardiovascular death was no longer significant (Table 3). However, when we controlled for $\triangle \mathrm{LDL}-\mathrm{C}$, the association of statin treatment with cardiovascular events remained significant, while the association with cardiovascular death was no longer significant (Table 4). Within the mediation analysis of cardiovascular events by $\triangle \mathrm{LR}$, the ACME and the ADE were significant in the treated group. This corresponded to $58 \%$ of the total effect in the statin-treated group mediated by $\Delta \mathrm{LR}$ for the Marschner covariate model. Similar results were observed when cardiovascular death was used as the outcome, with $61 \%$ of the total effect in the treatment group mediated by $\Delta \mathrm{LR}$ (Table 3 ).

When we performed the mediation analysis of cardiovascular events by $\triangle L D L-C$, there was no interaction effect, so the control and treatment groups are not reported separately. Within the combined groups, the ACME was not significant, but both the ADE and the total effect were significant. However, the average proportion mediated was only $31.8 \%$ and this was not significant. Similar, although weaker, effects were observed when cardiovascular death was considered the outcome (Table 4).

Stratification of the cohort based on $\triangle L R$. When the treatment group was stratified into quartiles of $\triangle L R$, we observed that the median $\Delta \mathrm{LR}$ within each of the 4 quartiles was $-1.81,-1.01,-0.54$, and 0.01 , respectively, with the median change in quartile $4(0.01)$ being close to 0 and similar to the median change of the placebo group (0.03). In contrast, all 4 quartiles of the treatment group showed a significant decrease in the median $\triangle$ LDL-C ( -1.29 to $-0.95 \mathrm{mM})$ compared with the median $\triangle \mathrm{LDL}-\mathrm{C}$ in the placebo group, which was close to 0 ( $-0.02 \mathrm{mM}$; Supplemental Figure 4$)$. The risk of cardiovascular events and cardiovascular death increased in higher quartiles of the lipid ratio compared with lower quartiles (Figure 4 and Supplemental Table 10). Q4 of the $\Delta \mathrm{LR}$ showed an increased risk, relative to the placebo group, for both cardiovascular events and cardiovascular death, which was independent of the $\triangle$ LDL-C (Figure 4).

Table 4. Mediation analysis of statin treatment on cardiovascular events and cardiovascular death by the change in LDL-C

\begin{tabular}{|c|c|c|c|c|}
\hline Effect $^{A}$ & Estimate $(95 \% \mathrm{CI})$ & $P$ value & Estimate $(95 \% \mathrm{CI})$ & $P$ value \\
\hline ACME $^{B}$ (average) & $-0.085(-0.202,0.040)$ & 1.69E-01 & $-0.050(-0.223,0.130)$ & $5.72 \mathrm{E}-01$ \\
\hline Total effect & $-0.266(-0.397,-0.140)$ & 1.00E-16 & $-0.241(-0.422,-0.070)$ & 6.20E-03 \\
\hline Prop. mediated ${ }^{\mathrm{D}}$ (average) & $31.8(-14.3,94.0)$ & 1.69E-01 & $20.7(-67.8,151.0)$ & 5.76E-01 \\
\hline Interaction test & $0.089(-0.130,0.312)$ & 4.29E-01 & $0.003(-0.325,0.343)$ & $9.81 \mathrm{E}-01$ \\
\hline
\end{tabular}




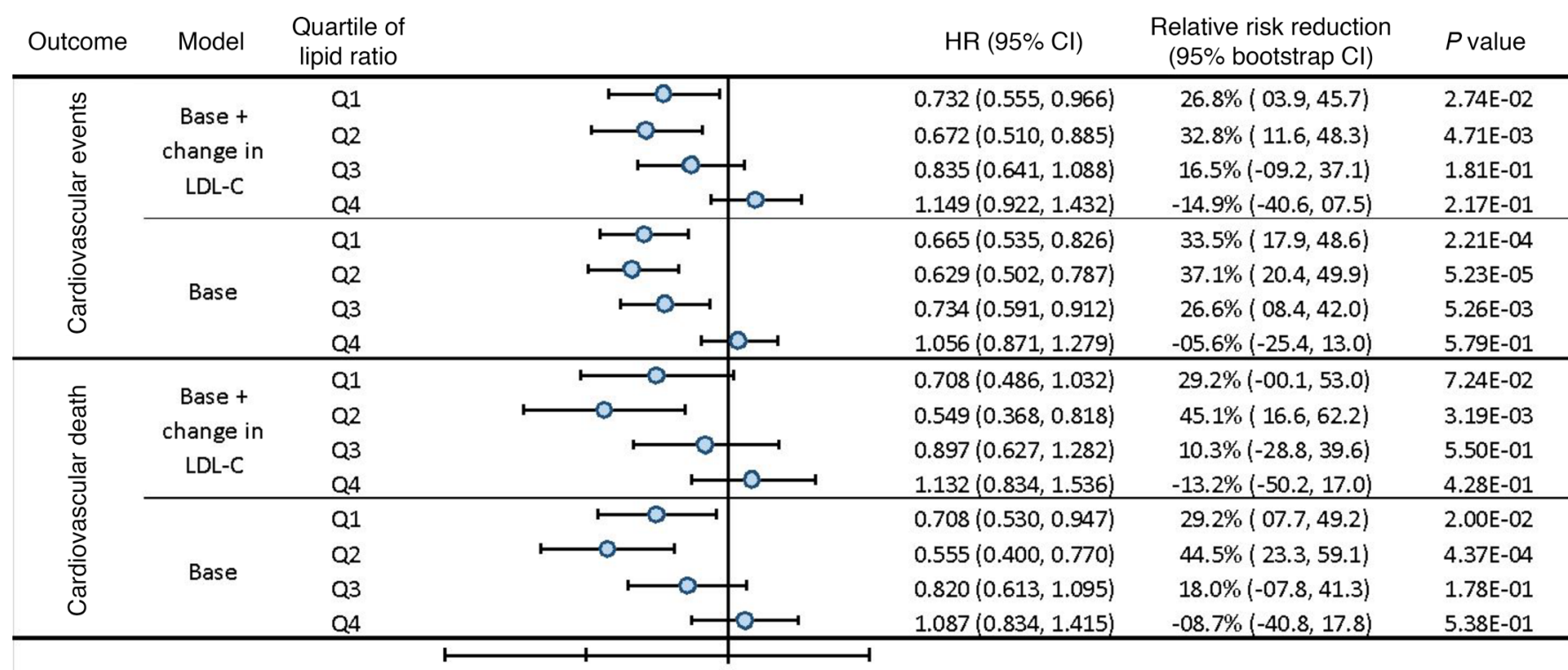

Figure 4. Association of treatment with future cardiovascular events and cardiovascular death in quartiles of $\Delta \mathrm{LR}, \mathrm{PI}(36: 2) / \mathrm{PC}(38: 4)$, in the statin treatment group $(\boldsymbol{n}=\mathbf{4 9 9 1 )}$. The samples in each quartile of the $\Delta L R$ on statin treatment were combined with all the samples in the placebo group, and a Cox regression analysis was performed to find the hazard ratio of treatment for cardiovascular events and death with or without adjustment for change in LDL-C. Base models included the Marschner covariates (age, sex, total cholesterol, HDL-C, current smoking, nature of prior acute coronary syndrome, revascularization, diabetes history, stroke history, and history of hypertension) previously developed in the LIPID cohort. Bootstrapping (1000 iterations) was performed to determine the Cls for the RRR.

\section{Discussion}

Statin treatment is widely administered to patients with or at high risk of cardiovascular disease, with the aim to control their LDL-C levels and thereby reduce incident cardiovascular events. Although the role of LDL-C in explaining the treatment effect of statins has been widely reported (14), the individual plasma lipid species that are also affected by statin treatment have not been studied in this context. In this study, we first characterized the change, resulting from statin treatment, in over 300 lipid species. We then investigated the relationship between these changes and cardiovascular outcomes and the effect on the RRR resulting from the pravastatin treatment. These analyses, based on change over 12 months, identified a lipid ratio, $\mathrm{PI}(36: 2) / \mathrm{PC}(38: 4)$, that stratified the population into those who experienced an RRR from pravastatin treatment and those who did not. Indeed, at least $25 \%$ of subjects received no benefit from pravastatin treatment in terms of reduced risk of cardiovascular events or death. These same lipids highlight metabolic pathways that may be important in mediating the protective effects of statins.

Pravastatin has differential effects on arachidonic acid and phosphatidylinositol metabolism. Although the phospholipid classes/subclasses, for the most part, showed only weak (small effect size) associations with statin treatment, at a species level, we observed 2 major effects of pravastatin. First, we observed that lipid species from multiple classes containing either arachidonic acid (20:4) or its biosynthetic precursor, dihomo- $\gamma$-linolenic acid (20:3), were positively associated with pravastatin treatment. Coupled with this was the observation that species containing linoleic acid (18:2) were typically negatively associated with pravastatin treatment, suggesting that the effect was driven by an increase in the conversion of linoleic acid to arachidonic acid. This was most obvious when we examined the association of pravastatin with change in the diacylglycerol species DG(18:0_18:2) (-6.064, $P=1.63 \mathrm{E}-06)$ and DG(18:0_20:4) (7.028, $P$ $=5.69 \mathrm{E}-06$; Supplemental Table 5), which demonstrates the opposing effects on the change in 18:2 and 20:4 fatty acids. Because this effect is evident in multiple lipid classes, including cholesteryl esters and several phospholipid species, it seems likely that this is controlled at the level of fatty acid metabolism and specifically the conversion of linoleic acid to arachidonic acid, which appears to be upregulated in response to statin treatment. Earlier reports have shown that $\delta-5$ desaturase, responsible for conversion of dihomo- $\gamma$-linolenic acid (20:3) to arachidonic acid (20:4), was upregulated by simvastatin in a manner dependent on SREBP-1 (15). This may then form part of a positive feedback loop because increased levels of polyunsaturated phosphatidylcholine species in the ER membrane have been reported to accelerate 
SREBP-1c cleavage via the SREBP cleavage-activating protein pathway (16), leading to nuclear localization and modulation of multiple lipid metabolic pathways.

In addition, 11 of 16 species of phosphatidylinositol were negatively associated with treatment, although these were offset by 2 abundant species, $\operatorname{PI}(38: 3)$ and $\operatorname{PI}(38: 4)$, that were positively associated, resulting in a weak negative association for the class. Phosphatidylinositol is synthesized by the enzyme CDP-diacylglycerol-inositol 3-phosphatidyltransferase, which transfers the phosphodiacylglycerol from the CDP-diacylglycerol onto a myoinositol. This is in contrast with other phospholipids, such as phosphatidylcholine, which is synthesized by the action of diacylglycerol cholinephosphotransferase, which transfers the phosphocholine group from CDP-choline onto a diacylglycerol. Thus it appears that in addition to altering the synthesis of arachidonic acid from linoleic acid, pravastatin may downregulate the production of phosphatidylinositol, possibly via CDP-diacylglycerol synthesis. This effect is compounded in the phosphatidylinositol species $\mathrm{PI}(36: 2)$ — which is primarily $\mathrm{PI}\left(18: 0 \_18: 2\right)$ with a small amount of $\mathrm{PI}(18: 1 / 18: 1)$ - as the decrease in availability of $18: 2$, resulting from the increased conversion to arachidonic acid, is compounded with the decrease in phosphatidylinositol synthesis.

Changes in lipid species in response to pravastatin treatment influences the $R R R$ for cardiovascular events. When we examined how the changes in these lipid species related to future cardiovascular events, we observed that when adjusted for the change in $\mathrm{PI}(36: 2)$ and $\mathrm{PC}(38: 4)$, the associations of treatment with cardiovascular events were weakened relative to models adjusted only for the $\triangle \mathrm{LDL}-\mathrm{C}$. This suggests that change in lipid species may provide an additional explanation for the statin treatment effect over and above the $\Delta$ LDL-C.

In our analyses, the decrease in the RRR resulting from the $\triangle \mathrm{LDL}-\mathrm{C}$ was less than that reported for the "on-treatment" LDL-C levels in the whole cohort (RRR of 25\% decreased to 13\%; ref. 17); however, the previous analysis considered only cardiovascular death and nonfatal myocardial infarction as the endpoint, whereas we have also included stroke as an endpoint. The more recent analysis of the LIPID study using the same cardiovascular outcomes and the $\triangle$ LDL-C as a covariate showed a change in the RRR from $24 \%$ to $15 \%$, which was closer to our results (4).

The positive association of the change in phosphatidylinositol species with cardiovascular outcomes, independent of traditional risk factors and $\triangle \mathrm{LDL}-\mathrm{C}$, indicates that a larger reduction in this lipid species lowers the risk of cardiovascular outcomes. However, in those species containing arachidonic acid, this relationship was negative. Thus, smaller reductions, or increases, in these lipid species were associated with fewer cardiovascular events. This may relate to the earlier analysis of the LIPID study where a reduction in lipoprotein phospholipase A2 activity resulted in a reduction of the pravastatin treatment effect from $23 \%$ to $10 \%$ (4); the arachidonic acid on the PC(38:4) would be a preferred substrate for the phospholipase A2, and so a decrease in the phospholipase A2 activity would lead to an increase in the $\mathrm{PC}(38: 4)$ and other arachidonic acid-containing species.

A lipid ratio identified those who did not receive a risk reduction from statin treatment. The opposing associations of these lipid species, which appeared to reflect multiple metabolic pathways, provided the opportunity to examine the lipid ratio $\mathrm{PI}(36: 2) / \mathrm{PC}(38: 4)$. We reasoned that the ratio of these lipids may capture the fatty acid metabolic pathway converting linoleic acid [18:2, present in $\mathrm{PI}(36: 2)]$ to arachidonic acid [20:4, present in $\mathrm{PC}(38: 4)$ ], in addition to other relevant pathways discussed above, and thereby provide a stronger signal relating pravastatin treatment and change in cardiovascular risk. The $\Delta \mathrm{LR}$ showed a stronger association with pravastatin treatment than the change in either individual species, independent of the change in clinical lipids $[\beta$-coefficient $=-26.0(P=3.21 \mathrm{E}-121)$; compared with $-14.6(P=8.10 \mathrm{E}-57)$ and $13.6(P=1.90 \mathrm{E}-69)$ in $\mathrm{PI}(36: 2)$ and $\mathrm{PC}(38: 4)$, respectively]. The $\Delta \mathrm{LR}$ was also a better predictor of both cardiovascular events and death than the individual species and had a larger effect on the RRR than either lipid species alone.

The mediation analysis suggests that $61 \%$ of the total effect of pravastatin treatment could be explained by an indirect action of the $\triangle \mathrm{LR}$. The significant interaction test and strong ACME in only the pravastatin group suggest the presence of a moderating effect of the $\Delta \mathrm{LR}$, more so than a mediating effect. Given the prospective study design and temporal sequence of events (statin treatment, then change in lipid ratio, and then reduced cardiovascular events), it is likely the $\Delta \mathrm{LR}$ provides a biomarker for the metabolic pathway(s) responsible for the protective effects of pravastatin treatment in this cohort. That is, individuals who were poor responders to pravastatin therapy had a smaller reduction in the lipid ratio and less protection against future cardiovascular events. Conversely, individuals who were positive responders to pravastatin therapy showed a greater reduction in the lipid ratio and, consequently, greater protection against future cardiovascular events. Earlier studies have indicated that a large part of the effect of pravastatin can be explained by 
changes in lipoprotein pools, particularly when total cholesterol and HDL-C are considered together (17). When we consider these results in the context of the reported action of simvastatin on arachidonic acid metabolism via a SREBP-1-dependent mechanism (15), it appears likely that the effect we are observing on lipid species and the lipid ratio may be a result of altered intracellular cholesterol metabolism signaling via SREBP-1 (or other signaling pathways) to alter lipid metabolism. These changes in lipid metabolism then exert a moderating effect on cardiovascular outcomes.

In light of these observations, we sought to assess whether this lipid ratio could predict who would benefit (or not benefit) from pravastatin treatment. Stratification of the treatment arm into quartiles of $\triangle \mathrm{LR}$ allowed us to demonstrate that $\mathrm{Q} 4$ showed no change in the lipid ratio upon pravastatin treatment but did show a marked (albeit slightly smaller) decrease in LDL-C (0.95 mM in Q4 compared with $1.29 \mathrm{mM}$ in Q1). Importantly, those individuals in Q4 showed no risk reduction for future cardiovascular events or cardiovascular death independent of the $\triangle \mathrm{LDL}-\mathrm{C}$. In contrast, Q1 and Q2 showed much greater risk reduction (up to $45 \%$ for cardiovascular death) than the overall risk reduction in the treatment arm $(21.6 \%$ for cardiovascular death). We also observed the same effect by simple calculation of the RRR based on event numbers (with no adjustment), thereby demonstrating the potential for translation of this lipid ratio into a clinical test to monitor statin therapy.

Lipid metabolic pathways may represent new therapeutic targets for prevention of cardiovascular events. These results demonstrate that pravastatin treatment has clinically important effects on lipid metabolism beyond LDL-C lowering and that these effects influence risk of future cardiovascular events.

Our ability to identify a subset of patients who did not receive a benefit from pravastatin treatment despite showing a reduction in LDL-C raises a number of questions related to patient management. Further lowering of LDL-C with higher doses or alternative therapies, such as PCSK9 inhibitors, may lead to both changes in the lipid ratio and reduced risk. Alternatively, new treatment strategies aimed at modulating the lipid metabolic pathways reflected by the lipid ratio may also have beneficial effects in this group of patients. Although no studies have been performed on phosphatidylinositol, there have been a number of studies to examine the association of different fatty acid species and cardiovascular event risk. These studies have reported mixed effects, but a meta-analysis of 10 studies showed a significant negative association of arachidonic acid with coronary outcomes (18). In 8 supplementation studies using primarily linoleic acid (18:2), which might be expected to increase the production of arachidonic acid, a trend toward a protective effect with a relative risk of 0.89 (CI, 0.71-1.12) was reported for the treatment groups (18). Further studies using arachidonic acid supplementation in secondary prevention will be required to test this concept.

Limitations of the study. Our findings are based on a post hoc analysis of a subcohort of the LIPID trial. Though the analysis represents the single largest lipidomic analysis performed to date, only half of the randomized patients have been used in this analysis and hence may not be fully representative of the actual trial. Furthermore, given that lipidomics was performed at the 1-year time point, patients who had an event in the first year have been excluded. The use of hazard ratios may have limited interpretability in a clinical setting in that they do not reflect the actual survival time of a patient. It also assumes proportionality of hazards, meaning that the ratio of hazard rates at each time interval is approximately constant during the study so that the predictive value of a biomarker is independent of time, which may not be realistic in clinical trials $(19,20)$ but is a reasonable working model.

In this study, we have used a lipid ratio, which provides a cumulative measure of different metabolic pathways; however, such measures may overestimate the effect size. The estimated change in RRR for pravastatin treatment accounted for by the change in lipid species may also be imprecise because of a number of possible measurement errors, including sample collection, assay sensitivity, and mass spectrometry measurements. To address these limitations, we calculated the statistical significance of the RRR change resulting from including the lipid species change in the base model, by randomizing cases within the treatment groups (10,000 replicates), and demonstrated that the effect of some lipid species and the lipid ratio were significantly different from the null hypothesis. We further performed formal mediation analyses to support our proposition that the lipid ratio mediated the effect of pravastatin on cardiovascular risk.

Finally, the results demonstrated here are based on pravastatin treatment, and so care must be taken in generalization to a class effect of statins. Although we did not have access to 2 independent statin intervention studies, we were able to validate the associations of statins on the plasma lipidome in a separate cohort from the ADVANCE study where $37 \%$ of participants were on a range of statin treatments, providing evidence for generalizability of these findings. Further, given the reported action of simvastatin on arachidonic 
acid metabolism via a SREBP-1-dependent mechanism (15), it seems likely that we are observing a class effect. Notwithstanding these observations, the results presented in this manuscript should be considered hypothesis generating, and their mechanistic links will require validation in independent cohorts as well as preclinical and clinical models.

In conclusion, this analysis represents the first large-scale longitudinal analysis of the effects of statins on plasma lipid species and the subsequent influence on cardiovascular risk. We have demonstrated that the change in phosphatidylinositol and phosphatidylcholine was associated with cardiovascular outcomes and resulted in a lower RRR independent of changes in LDL-C. The change in the ratio of 2 lipid species, $\mathrm{PI}(36: 2) / \mathrm{PC}(38: 4)$, after the initiation of pravastatin treatment moderated the RRR observed and identified at least $25 \%$ of the treatment group who did not receive a reduction in risk of future cardiovascular events. We propose that this lipid ratio may represent a useful biomarker to monitor the likely clinical benefits of statin treatment.

\section{Methods}

Study populations. The LIPID study design has been described in detail elsewhere (3). Subjects with a previous history of cardiovascular disease (myocardial infarction or hospital admission for unstable angina, $n=$ 9014), between 31 and 75 years of age, who had plasma total cholesterol level between 4.0 and $7.0 \mathrm{mmol} / \mathrm{L}$ and fasting triglycerides less than $5.0 \mathrm{mmol} / \mathrm{L}$, were randomized to pravastatin (40 $\mathrm{mg}$ daily) or placebo. The median follow-up period was 6 years. In the surviving patients, plasma samples and clinical lipid measurements were also collected at the first-year follow-up. Detailed lipidomic profiling was conducted on 4991 participants (49.7\% on pravastatin treatment) using both baseline and 1-year follow-up plasma samples (Supplemental Figure 1).

We used a second study, the ADVANCE case-cohort study, to validate the effect of statins on plasma lipid species. Details of the lipidomic analysis of the ADVANCE study have been published previously (7). Briefly, 342 lipid species in 3779 baseline samples from participants (37.4\% on statin treatment) were analyzed.

Lipid extraction and profiling. The extraction and lipidomic analysis method used in this study has been previously described $(7,21,22)$. Briefly, lipids were extracted from $10 \mu \mathrm{L}$ of human plasma using a single-phase butanol/methanol extraction, in batches of 486 - consisting of plasma samples, pooled plasma controls (every 20 samples), and water blanks (every 40 samples). To each sample, $100 \mu \mathrm{L}$ of the butanol/methanol (1:1) mix containing internal standards was added, and the samples were vortexed and sonicated in a water bath for 1 hour at $18^{\circ} \mathrm{C}-22^{\circ} \mathrm{C}$. Extracts were centrifuged $(16,000 \mathrm{~g}, 10$ minutes), and the supernatant was transferred to $0.2-\mathrm{mL}$ micro-inserts in sample vials for analysis. Lipidomic analysis was performed by liquid chromatography electrospray ionization tandem mass spectrometry on an Agilent 1290 liquid chromatography system combined with an Agilent 6490 triple quadrupole mass spectrometer, using Mass Hunter software (Agilent Technologies). Details are available in the Supplemental materials and Supplemental Table 11.

Statistics. Before statistical analysis, the concentration values for lipid species were normalized to the interquartile range, to facilitate the interpretation of the results from Cox regression models. The $P$ values were corrected for multiple comparisons using the Benjamini-Hochberg approach (23). $P$ values of less than 0.05 were considered significant. Analyses were performed using MATLAB (R2013a), Stata 13.1 (24), and R 3.3.2 (25).

To assess the effect of pravastatin on individual lipid classes, subclasses, and species, the mean percentage change of each lipid species from baseline to 1-year follow-up was compared between placebo and pravastatin treatment groups using Student's $t$ test. To further assess the relationship between treatment allocation and the percentage change in lipid concentration from baseline to 1-year follow-up, linear regression models were used, adjusting for either baseline age, sex, and BMI or for these covariates and the percentage change in total cholesterol, HDL-C, and triglycerides. Differences in lipid concentrations between the statin treatment and placebo groups were also determined at the 1-year time point (LIPID study) and at baseline (ADVANCE study) using linear regression models adjusted for age, sex, BMI, total cholesterol, HDL-C, and triglycerides to assess whether similar relationships were found.

To assess the association between change in each lipid species from baseline to the 1-year follow-up and future cardiovascular events and cardiovascular death, Cox regression models were used in a landmark analysis within the treatment group, the placebo group, and the whole cohort. The models were adjusted for age, sex, BMI, change in cholesterol, change in HDL-C, change in triglycerides, and baseline level of a given lipid species. 
The RRR (1-hazard ratio) resulting from pravastatin treatment was determined by a Cox regression model using treatment as a predictor and adjusting for covariates previously identified as the optimal set of baseline predictors associated with cardiovascular outcomes in the LIPID study (26): age, sex, total cholesterol, HDL-C, current smoking, nature of prior acute coronary syndrome, revascularization, diabetes history, stroke history, and history of hypertension (henceforth referred to as the "Marschner covariates"). To assess the effect of the change in lipid species on the RRR, the analysis was then repeated, adjusting for changes in LDL-C, changes in each lipid species, or both in addition to the baseline lipid levels and the interaction between change in lipid species and treatment. To assess the statistical significance of the change in the RRR obtained from these models, permutation tests were used (10,000 permutations). Briefly, the adjusted Cox regression model was repeated for each of the 10,000 permutations of the measurements of each lipid species within each group (pravastatin treatment and placebo). The change in RRR obtained from the original models for the inclusion of each lipid species was compared with the distribution of the RRR obtained after 10,000 individual permutations to obtain the $P$ value for the relative change in RRR ( $P$ value $=$ the fraction of values under the null hypothesis that are at least as extreme as the observed change in RRR from the model).

Based on the results of the analyses described above, the lipid species PI(36:2) and PC(38:4) were identified to exhibit marked but opposing relationships with statin treatment and cardiovascular outcomes. After validation of these associations with statin treatment in the ADVANCE study, the lipid ratio $\mathrm{PI}(36: 2) / \mathrm{PC}(38: 4)$ was investigated as a potentially sensitive marker of the RRR afforded by statin treatment. To assess this, the above analyses were repeated on the lipid ratio $\mathrm{PI}(36: 2) / \mathrm{PC}(38: 4)$.

To investigate whether the causal effect of the treatment on outcomes was mediated by the lipid ratio $\mathrm{PI}(36: 2) / \mathrm{PC}(38: 4)$, we performed causal mediation analysis (27). Analyses were conducted using either the change in lipid ratio $\mathrm{PI}(36: 2) / \mathrm{PC}(38: 4)(\Delta \mathrm{LR})$ or change in LDL-C ( $\triangle \mathrm{LDL}-\mathrm{C})$ as the mediator. Briefly, we first determined the association of statin treatment with cardiovascular events and cardiovascular death using Cox regression adjusted for the Marschner covariates. We then determined the association of the $\triangle \mathrm{LR}$ and $\triangle \mathrm{LDL}-\mathrm{C}$ with statin treatment using linear regression models adjusted for the Marschner covariates. With both of these conditions satisfied, causal mediation analysis then estimated the proportion of risk in the outcome model explained by a direct effect of statins on cardiovascular outcomes - the ADE - and the proportion that was mediated by $\triangle \mathrm{LR}$ or $\triangle \mathrm{LDL}-\mathrm{C}$ - the ACME (Supplemental Figure 2; ref. 28). To test for differences in the ACME depending on treatment status, an interaction term was introduced between the treatment and the outcome. The mediation package allowed resampling procedures to estimate these parameters and their CIs; hence, 10,000 bootstraps were used to derive the CIs of the ADE and ACME described above.

Finally, the LDL-C lowering and the RRR by pravastatin treatment were explored in quartiles of the lipid ratio in the statin treatment group compared with the placebo group. In this analysis, the samples in each quartile of the lipid ratio within the treatment group were pooled together with the entire placebo group, and a Cox regression model, adjusted for Marschner covariates, was used to find the RRR by pravastatin treatment. RRR was also calculated based on the relative risk, without adjusting for covariates, where $R R R=$ (relative risk of placebo group - relative risk of treatment group)/relative risk of placebo group.

Study approval. All participants of the LIPID and ADVANCE studies provided written informed consent, and the studies were approved by the University of Sydney Human Research Ethics Committee. The analysis of archived plasma samples presented here was approved by the Alfred Hospital Ethics Committee.

\section{Author contributions}

KSJ, PAM, CG, EHB, AK, MW, ICM, GW, BAK, and PJM developed the statistical analysis protocols and performed the analysis. CKB, ZHA, NAM, KH, MJM, and PJM developed the mass spectrometry protocols, performed the experiments, and generated the data. PJN, EHB, AK, PT, DRS, JS, and AMT oversaw the LIPID trial and provided key inputs on interpretation of the data. SZ, GSH, JC, and MW oversaw the ADVANCE trial and provided key input on interpretation of the data. KSJ, PAM, CG, and PJM wrote the manuscript. 


\section{Acknowledgments}

The LIPID study was supported by Bristol-Myers Squibb and the NHMRC (grants 211086, 358395). JC and MW are supported by an NHMRC program grant (1149987) and MW by an NHMRC fellowship (108026). This study was supported by an NHMRC grant (1029754) and the Operational Infrastructure Support Program of the Victorian government of Australia. We would like to thank the investigators of the LIPID and the ADVANCE studies and the patients who participated in these studies. We acknowledge Michelle Cinel and Ricardo Tan for their technical support in the lipidomic analyses. See the Supplemental Acknowledgments for the LIPID Study Investigators' details.

Address correspondence to: Peter J. Meikle, Baker Heart and Diabetes Institute, 75 Commercial Road, Melbourne, Victoria 3004, Australia. Phone: 61.0.3.8532.1770; Email: peter.meikle@baker.edu.au.

1. Scott R. Lipid modifying agents: mechanisms of action and reduction of cardiovascular disease. Clin Exp Pharmacol Physiol. 1997;24(5):A26-A28.

2. Cholesterol Treatment Trialists' (CTT) Collaborators, et al. The effects of lowering LDL cholesterol with statin therapy in people at low risk of vascular disease: meta-analysis of individual data from 27 randomised trials. Lancet. 2012;380(9841):581-590.

3. Long-Term Intervention with Pravastatin in Ischaemic Disease (LIPID) Study Group. Prevention of cardiovascular events and death with pravastatin in patients with coronary heart disease and a broad range of initial cholesterol levels. NEngl J Med. 1998;339(19):1349-1357.

4. White $\mathrm{HD}$, et al. Changes in lipoprotein-associated phospholipase A2 activity predict coronary events and partly account for the treatment effect of pravastatin: results from the Long-Term Intervention with Pravastatin in Ischemic Disease study. J Am Heart Assoc. 2013;2(5):e000360.

5. Olsson AG, et al. High-density lipoprotein, but not low-density lipoprotein cholesterol levels influence short-term prognosis after acute coronary syndrome: results from the MIRACL trial. Eur Heart J. 2005;26(9):890-896.

6. Puri R, et al. C-reactive protein, but not low-density lipoprotein cholesterol levels, associate with coronary atheroma regression and cardiovascular events after maximally intensive statin therapy. Circulation. 2013;128(22):2395-2403.

7. Alshehry $\mathrm{ZH}$, et al. Plasma lipidomic profiles improve on traditional risk factors for the prediction of cardiovascular events in type 2 diabetes mellitus. Circulation. 2016;134(21):1637-1650.

8. Laaksonen R, et al. Plasma ceramides predict cardiovascular death in patients with stable coronary artery disease and acute coronary syndromes beyond LDL-cholesterol. Eur Heart J. 2016;37(25):1967-1976.

9. Mundra PA, et al. Large-scale plasma lipidomic profiling identifies lipids that predict cardiovascular events in secondary prevention. JCI Insight. 2018;3(17):121326.

10. Stegemann C, et al. Lipidomics profiling and risk of cardiovascular disease in the prospective population-based Bruneck study. Circulation. 2014;129(18):1821-1831.

11. Tarasov K, et al. Molecular lipids identify cardiovascular risk and are efficiently lowered by simvastatin and PCSK9 deficiency J Clin Endocrinol Metab. 2014;99(1):E45-E52.

12. Meikle PJ, et al. Statin action favors normalization of the plasma lipidome in the atherogenic mixed dyslipidemia of MetS: potential relevance to statin-associated dysglycemia. J Lipid Res. 2015;56(12):2381-2392.

13. Ng TW, et al. Dose-dependent effects of rosuvastatin on the plasma sphingolipidome and phospholipidome in the metabolic syndrome. J Clin Endocrinol Metab. 2014;99(11):E2335-E2340.

14. Deedwania P, et al. Reduction of low-density lipoprotein cholesterol in patients with coronary heart disease and metabolic syndrome: analysis of the Treating to New Targets study. Lancet. 2006;368(9539):919-928.

15. Risé P, Ghezzi S, Carissimi R, Mastromauro F, Petroni A, Galli C. $\delta 5$ Desaturase mRNA levels are increased by simvastatin via SREBP-1 at early stages, not via PPAR $\alpha$, in THP-1 cells. Eur J Pharmacol. 2007;571(2-3):97-105.

16. Rong X, Wang B, Palladino EN, de Aguiar Vallim TQ, Ford DA, Tontonoz P. ER phospholipid composition modulates lipogenesis during feeding and in obesity. J Clin Invest. 2017;127(10):3640-3651.

17. Simes RJ, et al. Relationship between lipid levels and clinical outcomes in the Long-term Intervention with Pravastatin in Ischemic Disease (LIPID) Trial: to what extent is the reduction in coronary events with pravastatin explained by on-study lipid levels? Circulation. 2002;105(10):1162-1169.

18. Chowdhury R, et al. Association of dietary, circulating, and supplement fatty acids with coronary risk: a systematic review and meta-analysis. Ann Intern Med. 2014;160(6):398-406.

19. Blagoev KB, Wilkerson J, Fojo T. Hazard ratios in cancer clinical trials - a primer. Nat Rev Clin Oncol. 2012;9(3):178-183.

20. Spruance SL, Reid JE, Grace M, Samore M. Hazard ratio in clinical trials. Antimicrob Agents Chemother. 2004;48(8):2787-2792.

21. Alshehry ZH, Barlow CK, Weir JM, Zhou Y, McConville MJ, Meikle PJ. An efficient single phase method for the extraction of plasma lipids. Metabolites. 2015;5(2):389-403.

22. Weir JM, et al. Plasma lipid profiling in a large population-based cohort. J Lipid Res. 2013;54(10):2898-2908.

23. Benjamini Y, Hochberg Y. Controlling the false discovery rate: a practical and powerful approach to multiple testing. $J R$ Stat Soc Ser B. 1995;57(1):289-300.

24. Stata Corp. College Station, Texas: StataCorp LP; 2013.

25. R Core Team. R Foundation for Statistical Computing, Vienna, Austria; 2016.

26. Marschner IC, et al. Long-term risk stratification for survivors of acute coronary syndromes. Results from the Long-term Intervention with Pravastatin in Ischemic Disease (LIPID) Study. LIPID Study Investigators. J Am Coll Cardiol. 2001;38(1):56-63.

27. Tingley D, Yamamoto T, Hirose K, Keele L, Imai K. Mediation: R package for causal mediation analysis. Journal of Statistical Software. 2014;1(5).

28. Imai K, Keele L, Tingley D. A general approach to causal mediation analysis. Psychol Methods. 2010;15(4):309-334. 